\title{
Possible Isostructural Transitions in the Ferroelectric Liquid Crystals in High External Electric Fields
}

\author{
Brigita Urbanc \\ Center for Polymer Studies and Department of Physics, Boston \\ University, Boston, USA

\section{Mojea Čepič} \\ Jožef Stefan Institute, Ljubljana, Slovenia; Faculty of Education, \\ University of Ljubljana, Ljubljana, Slovenia and Faculty of Education, \\ University of Primorska, Koper, Slovenia
}

Two different types of isostructural transitions between two homogeneously tilted ferroelectric liquid crystalline structures are discussed. A strong biquadratic coupling between the tilt and the polarization leads to the discontinuous jump of the tilt magnitude when the electric field is increased. A strong anisotropic quadratic coupling with the electric field leads to the continuous structural transition associated with a change of the direction of the tilt.

Keywords: ferroelectric liquid crystals; isostructural transition

\section{INTRODUCTION}

Ferroelectric liquid crystals discovered in 1974 [1] have been extensively studied since [2]. Due to their polar properties they can be easily manipulated by external electric field. Although phase diagrams in the electric fields have been experimentally studied [3,4] and theoretically explained [5], recent observations reopened some of presumably already solved problems [6].

When external electric field is applied to a ferroelectric liquid crystal, two different behaviours are observed. In the non-tilted Sm A* phase, the field-imposed ordering of the molecular polarizations induces a homogeneous molecular tilt perpendicular to the electric

The authors are very grateful to Z. Kutnjak and P. Keyes for stimulating discussions.

Address correspondence to Mojca Čepič, Jožef Stefan Institute, Jamova 39, Ljubljana 1000, Slovenia. E-mail: mojca.cepic@ijs.sl 
field as a consequence of the linear coupling between the tilt and the polarization. This phenomenon is called the electroclinic effect. The chiral tilted $\mathrm{Sm} \mathrm{C}^{*}$ phase is helicoidally modulated. In an external electric field the modulation deforms, its period increases, and the structure unwinds into the homogeneously tilted $\mathrm{Sm} \overline{\mathrm{C}}^{*}$ phase. Both phases have the same macroscopic symmetry as the achiral homogeneously tilted Sm C phase, but they are stable only in the presence of the external electric field. However, recent experimental evidence showed that an additional structural transition seems to exist in the region of homogeneously tilted ferroelectric structure induced by the external electric field. In this contribution we show that two types of isostructural transitions between the two homogeneously tilted structures exist at least theoretically. If the biquadratic coupling of the tilt and the polarization is significant, the discontinuous change of the tilt magnitude occurs at a critical electric field. On the other hand, if the anisotropic quadratic coupling with the electric field is important, the continuous transition where the tilt changes its direction with respect to the direction of the external electric field is possible.

\section{THE FREE ENERGY}

The free energy of the ferroelectric liquid crystals was first introduced by Pikin and Indebom [7]. As the aim of this paper is a theoretical prediction of the existence of the isostructural transition between two homogeneously tilted phases, induced by an external homogeneous electric field, we consider only the simplest form of the free energy expansion. We take into account both order parameters, tilt $\vec{\xi}=\left\{\xi_{x}, \xi_{y}\right\}$ and polarization $\vec{P}=\left\{P_{x}, P_{y}\right\}$, and the electric field along the $y$ direction $\vec{E}=\{0, E\}$ :

$$
F=\frac{1}{2} a_{0} \xi^{2}+\frac{1}{4} b_{0} \xi^{4}+\frac{1}{2 \varepsilon^{*}} P^{2}+c_{p}(\vec{P} \times \vec{\xi})_{z}-\vec{P} \cdot \vec{E}
$$

Here only the first parameter $a_{0}=\alpha\left(T-T_{0}\right)$ is temperature dependent and changes the sign at the temperature $T_{0}$. This temperature corresponds to the transition temperature to the tilted phase in a racemic system in the absence of external fields where no polarization and chiral interactions are present. For systems with continuous phase transitions in the absence of external fields, the parameter $b_{0}$ is positive. The electrostatic free energy of ordered dipoles is given by positive constant $\varepsilon^{*}$ as the polarization $P$ is not a proper order parameter. It is induced by the tilt due to the piezoelectric coupling given by $c_{p}$. 
We do not consider nonhomogeneities induced by chiral interactions and defects in the free energy expansion [7], therefore the terms related to derivatives of order parameters, are not included.

\section{BIQUADRATIC COUPLING}

The following terms are added to the free energy Eq. (1), to analyze the behaviour of the system in presence of additional interactions, that are required by the symmetry arguments [8].

$$
-\frac{1}{2} \Omega(\vec{P} \times \vec{\xi})^{2}+\frac{1}{4} \eta P^{4}+\frac{1}{6} c_{0} \xi^{6}
$$

The first term $\Omega$ resumes the fact that typical molecules are lath-like objects and they, in general, order quadrupolarly. This effect may significantly reduce electrostatic dipolar contribution and the polarization ordering may become critical. Therefore the fourth order term in the polarization $\eta$ is included. A number of experiments have shown that the tilt dependence on the temperature is close to the tricritical behaviour in many materials [2], but we consider here the sixth order term in the tilt $c_{0}$ also due to theoretical reasoning. The quadrupolar term $\Omega$ favours structures with a large tilt and polarization. If so, the tilt should be additionally stabilized to account for general conditions with the saturated tilt almost always found in ferroelectric liquid crystals at lower temperatures. In the unwound ferroelectric liquid crystal the situation is straightforward. The polarization $\vec{P}$ of all layers is oriented parallel to the electric field and the homogeneous tilt of the magnitude $\theta$ is everywhere perpendicular to it. The free energy is

$$
G=\frac{1}{2} a_{0} \theta^{2}+\frac{1}{4} b_{0} \theta^{4}+\frac{1}{6} c_{0} \theta^{6}+\frac{1}{2 \varepsilon^{*}} P^{2}+\frac{1}{4} \eta P^{4}-c_{p} \theta P-P E-\Omega \theta^{2} P^{2}
$$

and has to be minimized with respect to both: the magnitude of the tilt and the magnitude of the polarization. The results of the minimization are given in Figure 1. Minimization equations are solved numerically.

Above the transition temperature to the tilted phase, the initial non-tilted Sm A structure becomes slightly tilted due to the electroclinic effect. On further increase of the external electric field, the tilt changes anomalously, Figure 1a. The linear electroclinic effect can be observed only for weak fields. The anomalous change of the tilt 

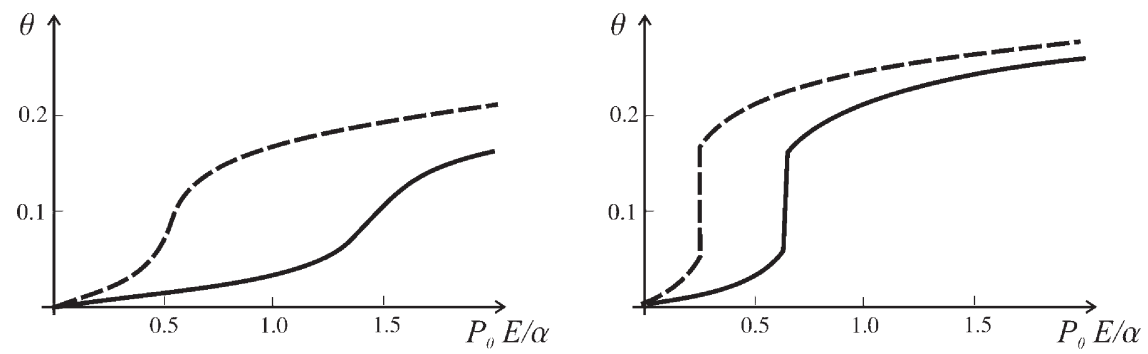

FIGURE 1 The tilt dependence on electric field calculated for the set of model parameters expressed in units of $\alpha: b_{0}=-10, c_{0}=1000, c_{p}=0.1, \varepsilon^{*}=1$, $\eta=50$. Solid line: $T-T_{0}=2$, dashed line: $T-T_{0}=1$; (a) $\Omega=20$; (b) $\Omega=40$.

crosses over to a discontinuous change of the tilt magnitude at a strong biquadratic coupling. Both structures have the same symmetry and the transition is similar to the liquid-vapour transition observed in water, where only a density changes discontinuously while the symmetry properties of both phases remain the same.

\section{QUADRATIC COUPLING WITH THE ELECTRIC FIELD}

At higher electric fields another possible reason for the structural change of the homogeneously tilted ferroelectric liquid crystal, could be a quadratic dielectric coupling with the external electric field. The effect of the anisotropy of the molecules with respect to the effect of the molecular dipole moment becomes more important for stronger electric fields. Both interactions - the linear and the quadratic coupling - compete. The linear coupling favours tilts perpendicular to the electric field, while at positive anisotropies that are typical for elongated molecules at low frequencies, parallel or antiparallel tilt alignments are favourable. Therefore, the change in the direction of the tilt can be expected at a critical electric field.

We consider anisotropic quadratic coupling with the electric field separately from the biquadratic coupling between the tilt and polarization analyzed in the previous section to get insights into possible mechanisms that would induce such structural changes. The quadratic coupling with the electric field is added to the free energy (1)

$$
-\frac{1}{2}\left(\varepsilon_{e}-\varepsilon_{o}\right) \varepsilon_{0}(\vec{E} \cdot \vec{\xi})^{2} .
$$

Here the $\varepsilon_{e}$ and $\varepsilon_{o}$ are the larger and the smaller value of the uniaxial dielectric tensor. The homogeneous Ansatz, in which polarization is 
perpendicular to the tilt,

$$
\begin{aligned}
& \vec{\xi}=\theta\{\cos \phi, \sin \phi\} \\
& \vec{P}=P\{-\sin \phi, \cos \phi\}
\end{aligned}
$$

where the angle $\phi$ is measured with respect to the $x$-axis. After solving for the polarization, we get

$$
G=\frac{1}{2} \tilde{a}_{0} \theta^{2}+\frac{1}{4} b_{0} \theta^{4}-\varepsilon^{*} c_{p} \theta E \cos \phi-\frac{1}{2}\left(\varepsilon_{e}-\varepsilon_{o}\right) \varepsilon_{0} E^{2} \theta^{2} \sin ^{2} \phi
$$

and

$$
\tilde{a}_{0} \theta^{2}=\alpha\left(T-T_{0}\right)-\varepsilon^{*} c_{p}^{2} .
$$

Minimization with respect to the tilt direction $\phi$ leads to two possible solutions

$$
\begin{aligned}
& \phi=0 \text { for } E<E_{c} \\
& \phi=\arccos \left(\frac{\varepsilon^{*} c_{p}}{\left(\varepsilon_{e}-\varepsilon_{o}\right) \varepsilon_{0} \theta E}\right) \text { for } E>E_{c}
\end{aligned}
$$

and the critical field

$$
E_{c}=\frac{\varepsilon^{*} c_{p}}{\left(\varepsilon_{e}-\varepsilon_{o}\right) \varepsilon_{0} \theta} .
$$

Above the critical field $E_{c}$, the tilt is rotated by an angle $\phi$ toward parallel or antiparallel orientation with respect to the direction of the external field, Figure 2. The transition is continuous and both orientations $\phi>0$ and $\phi<0$ are equally favourable. Therefore, we expect that

(a)

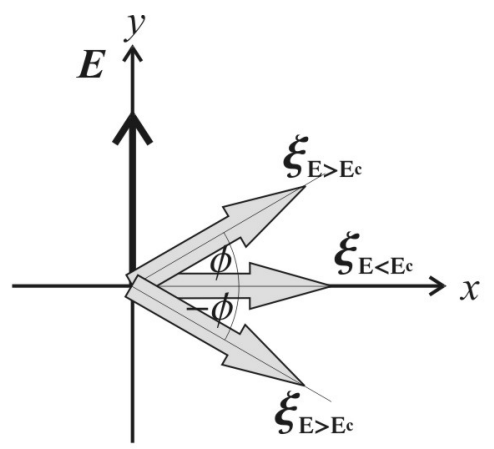

(b)

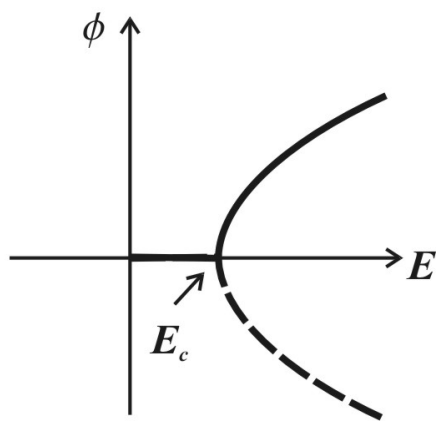

FIGURE 2 (a) Schematic presentation of the geometry of the problem. (b) Schematic dependence of the tilt direction. 
two types of the domains with different optical properties are formed simultaneously. The formation of domains can be in principle observed electrooptically. Considering Eq. (9), we can guess what types of the materials would be the most appropriate for the study. The critical electric field $E_{c}$ is proportional to the polarization $P$. The magnitude of the polarization $P$ is proportional to the piezoelectric parameter of chiral character, $c_{p}$. Racemization of the sample would lower the critical field and the phenomenon might become observable under these conditions. The critical field $E_{c}$ is also inversely proportional to the magnitude of the tilt. Therefore, we can expect that $E_{c}$ would be observed in materials with large tilt and at lower temperatures.

\section{CONCLUSIONS}

The two types of possible isostructural transitions, i.e., the transitions between the two structures of different order but equal symmetries, are reported.

The biquadratic coupling, if present in the system, is the reason for the anomalous electroclinic effect and can, for strong couplings $\Omega$, lead to a discontinuous change of the tilt magnitude. The phenomenon could be observed above the transition temperature to the tilted phase.

The competing mechanism between the linear and the quadratic coupling with the external electric field is the reason for a continuous transition from the structure with a tilt perpendicular to the electric field, to the structure with a general angle of the tilt. The appearance of the two different domains with a tilt direction forming and an angle $\phi$ or $-\phi$ with respect to the $x$ axis should be detected electrooptically. To the best of our knowledge, the only experiment that gives a hint of possible behaviour of one or both of the reported types is the reference [6], which actually stimulated the present work.

\section{REFERENCES}

[1] Meyer, R. B., Liebert, L., Strzelecki, L., \& Keller, P. (1975). J. Phys. Lett., 36, L69.

[2] Muševič, I., Blinc, R., \& Žekš, B. (2000). The Physics of Ferroelectric and Antiferroelectric Liquid Crystals, World Scientific: Singapore.

[3] Dumrongrattana, S. \& Huang, C. C. (1986). J. Phys (Paris), 47, 2117.

[4] Levstik, A., Kutnjak, Z., Žekš, B., Dumrongrattana, S., \& Huang, C. C. (1991). J. Phys. II (France), 1, 797.

[5] Urbanc, B. K. \& Žekš, B. (1995). Phys. Rev. E, 51, 1569.

[6] Ghodoussi, F., Pantea, M., Keyes, P., Naik, R., \& Vaishnava, P. (2003). Phys. Rev. E, 68, 051706.

[7] Pikin, S. A. \& Indebom, V. L. (1978). Ferroelectrics, 20, 151.

[8] Žekš, B. (1984). Mol. Cryst. Liq. Cryst., 114, 259. 